\title{
THE EXISTENCE AND UNIQUENESS OF THE SOLUTION FOR A CLASS OF MIXED-TYPE INTEGRAL EQUATION
}

\section{Zuolun Zhao ${ }^{\mathrm{a}}$ and Xinfeng Wang $\mathrm{b}^{*}$}

aNo. 1 Middle School, Dongying District, Shandong, 257091, China

${ }^{\mathrm{b}}$ Basic Course Department of Beijing Union University, Beijing, 100101, China

\begin{abstract}
By the generalization of Darbo's fixed point theorem, the existence and uniqueness theorem about the solutions of nonlinear mixed Volterra-Fredholm integral equation in Banach spaces has been established. Moreover, an iterative sequence converging to our unique solution has been constructed and an error estimate formula is given. Finally, applying those to three-point boundary value problems of second order integro-differential equations, some new conclusion about its solutions has been obtained.
\end{abstract}

Keywords: mixed-type integral equation, three-point boundary value problem, Banach space, fixed point.

${ }^{*}$ Corresponding author.

E-mail address: wangxf728@263.net (Xinfeng Wang).

Copyright ( 2015 Scientific Advances Publishers

2010 Mathematics Subject Classification: 45J05, 34K30.

Submitted by Jianqiang Gao.

Received April 1, 2015 


\section{Introduction}

The integral equation, since many of the mathematical problems and physical problem come down to it, has always been an active field. The study aiming to them emerge in an endless stream.

Over the years, many paper investigate the Volterra or Fredholm integral equations on the real space $R$ or $R^{n}$ [1], [2], [3]. Recently, in Banach spaces, Long and Nelakantib [4] investigate the iterative methods of the linear Fredholm integral equations, Li and Wang [5] discuss the existence of multiple solutions for the nonlinear Fredholm integral equations

$$
x(t)=\int_{a}^{b} H(t, s, x(s)) d s
$$

and Qian and Zhao [6] obtained the coupled quasi-solutions and solutions of nonlinear impulsive Fredholm integral equations:

$$
x(t)=x_{0}(t)+\lambda \int_{t_{0}}^{T} H(t, s, x(s)) d s+\sum_{t_{0}<t_{k}<t} a_{k}(t) I_{k}\left(x\left(t_{k}\right)\right) .
$$

For discussing the boundary value problem (BVP) of the differential or integro-differential equation, the peoples often change it into an equivalent integral equation. Relying on the integral equations, Chen and $\mathrm{Li}$ [7] has discussed three-point boundary value problems in Banach spaces

$$
\left\{\begin{array}{l}
u^{\prime \prime}(t)+f\left(t, u(t), u^{\prime}(t)\right)=\theta, \quad 0<t<1 \\
u(0)=\theta, \quad u(1)=b u(\mu)
\end{array}\right.
$$

and obtain the existence of at least one positive solution. By this method, Wang et al. [8] study the two-point boundary value problems, Liu et al. [9] and Feng and Pang [10] investigate for impulsive two-point and threepoint boundary value problems, respectively, and obtain the existence of their positive solutions. 
In this paper, we study the following integral equation:

$$
u(t)=\psi(t)+\int_{0}^{a} W(t, s) f(s, u(s), T u(s),(S u)(s)) d s
$$

where $W(\cdot, t), W(t, \cdot) \in C[0, a], \psi \in C[0, a], T u=\int_{0}^{t} k(t, s) u(s) d s, S u=\int_{0}^{a} h(t, s) u$ $(s) d s, h(t, s) \in C(J \times J, R), k(t, s) \in C(D, R), D=\{(t, s) \in J \times J: t \geq s\}$ and the same as follows. The existence of solutions of (1.1) is obtained. And in another proper conditions, the unique solution of (1.1) and an error estimate of the solution are gotten. Finally, we applied it to the three-point boundary value problem (BVP):

$$
\left\{\begin{array}{l}
u^{\prime \prime}(t)=f(t, u(t), T u(t), S u(t)), \quad \forall t \in J=[0, a], \\
u(0)=0, \quad u(a)=b u(\mu),
\end{array}\right.
$$

where $\mu \in(0, a)$. And get some new results. As follows, let $k_{0}=\max$ $\{|k(t, s)|:(t, s) \in D\}, h_{0}=\max \{|h(t, s)|:(t, s) \in J \times J\}$.

\section{Preliminaries and Some Lemmas}

Let $\alpha(\cdot)$ denotes the Kuratowski measure of non-compactness in Banach space $E$. For details on the definition and properties of the measure of non-compactness, please refer to the references [11], [12], [13].

Lemma 2.1 ([12]). If $H \subset C(J, E)$ is bounded and equicontinuous, then

$$
\alpha(H)=\max _{t \in J} \alpha(H(t)) .
$$

Lemma 2.2 ([13]). If $H \subset C(J, E)$ is bounded and the elements of $H$ are equicontinuous on $J$, then $\alpha(\{u(t) \mid u \in H\})$ is continuous on $J$ and

$$
\alpha\left(\left\{\int_{J} u(t) d t \mid u \in H\right\}\right) \leq \int_{J} \alpha(\{u(t) \mid u \in H\}) d t .
$$


Lemma 2.3 ([14]). Let $F$ be a closed and convex subset of a real Banach space $E$, the operator $A: F \rightarrow F$ be continuous and $A(F)$ be bounded. For any bounded subset $H \subset F$, set

$$
A^{1}(H)=A(H), \quad A^{n}(H)=A\left(\bar{c} o\left(A^{n-1}(H)\right)\right), \quad n=2,3, \ldots .
$$

If there exist a constant $0 \leq \gamma<1$ and a positive integer $n_{0}$ such that for any bounded subset $H \subset F$

$$
\alpha\left(A^{n_{0}}(H)\right) \leq \gamma \alpha(H),
$$

then $A$ has at least one fixed point in $F$.

Lemma 2.4 ([15]). Assume for all $r>0, f$ is bounded and uniformly continuous on $J \times B_{r} \times B_{r} \times B_{r} \times B_{r}$ and $H \subset C(J, E)$ is bounded and equicontinuous, then $\{f(t, x(t),(T x)(t),(S x)(t)): x \in H\}$ is bounded and equicontinuous in $C(J, E)$.

\section{Main Results}

For convenience, we give the assumptions as follows:

(H1). For any $r>0, f$ is bounded and uniformly continuous on $J \times B_{r} \times B_{r} \times B_{r}$, and there exist non-negative Lebesgue integrable functions $L_{i}(i=1,2,3)$ such that for any bounded sets $B_{i} \in B_{r}(i=1,2,3)$ and $t \in J$,

$$
\alpha\left(f\left(t, B_{1}, B_{2}, B_{3}\right)\right) \leq L_{1}(t) \alpha\left(B_{1}\right)+L_{2}(t) \alpha\left(B_{2}\right)+L_{3}(t) \alpha\left(B_{3}\right) .
$$

(H2). $\beta=\limsup _{\|x\|+\|u\|+\|v\| \rightarrow+\infty}\left(\sup _{t \in J} \frac{\|f(t, x, u, v)\|}{\|x\|+\|u\|+\|v\|}\right), k_{0}=\max \{|k(t, s)|:(t, s) \in D\}$, $h_{0}=\max \{|h(t, s)|:(t, s) \in J \times J\}$ such that

$$
\eta \beta<1
$$

where $\eta=\max \left\{\max _{t \in[0, a]} \int_{0}^{a} W(t, s)\left[\left(1+s k_{0}+h_{0}\right) d s, \max _{t \in[0, a]} \int_{0}^{a} W_{t}^{\prime}(t, s)(1+\right.\right.$ $\left.\left.s k_{0}+h_{0}\right) d s\right\}$. 
(H3). There exists non-negative function $\sigma, \omega \in C[0,1]$ such that

$$
|W(t, s)| \leq \sigma(t) \omega(s), \quad \forall t, s \in[0,1]
$$

and

$$
\rho=\int_{0}^{1} \sigma(s) \omega(s)\left[L_{1}(s)+s k_{0} L_{2}(s)+a h_{0} L_{3}(s)\right] d s<1
$$

Theorem 3.1. Let $E$ be a real Banach space, the assumptions (H1)-(H3) be satisfied, then the mixed-type integral equation (1.1) has at least one solution $u^{*} \in C(J, E)$.

Proof. Let

$$
(A u)(t)=\psi(t)+\int_{0}^{a} W(t, s) f(s, u(s), T u(s),(S u)(s)) d s .
$$

We will prove the operator $A$ has fixed point on $C(J, E)$.

By (3.1), there exists $\beta^{\prime}>\beta$ such that

$$
\eta \beta^{\prime}<1
$$

From the definitions of $\beta$ and $\beta^{\prime}$, there exists $N>0$ such that

$$
\|f(t, x, u, v)\|<\beta^{\prime}(\|x\|+\|u\|+\|v\|), \quad \forall t \in J, \quad\|x\|+\|u\|+\|v\|>N .
$$

Since $f$ is bounded on any bounded set, we have

$$
\|f(t, x, u, v)\| \leq \beta^{\prime}(\|x\|+\|u\|+\|v\|)+M, \quad \forall t \in J, \quad x, u, v \in E, \text { (3.6) }
$$

where $M=\sup \{\|f(t, x, u, v)\|: t \in J,\|x\|+\|u\|+\|v\|<N\}<\infty$. For any $u \in C(J, E)$ and $t \in J$, it follows from (3.4) and (3.6)

$$
\begin{aligned}
\|(A u)(t)\| & \leq\|\psi(t)\|+\int_{0}^{a}\|W(t, s) f(s, u(s), T u(s),(S u)(s))\| d s \\
& \leq\|\psi(t)\|+\beta^{\prime} \int_{0}^{a}|W(t, s)|\left[\left(1+s k_{0}+h_{0}\right)\|u(s)\|+M\right] d s \\
& \leq \eta \beta^{\prime}\|u\|_{C}+K .
\end{aligned}
$$


Let $r>K /\left(1-\eta \beta^{\prime}\right), T_{r}=\left\{x \in C(J, E):\|x\|_{C} \leq r\right\}$. Since $f$ is uniformly continuous, we have that $A: T_{r} \rightarrow T_{r}$ is continuous and bounded.

Let $F=\bar{c} o A\left(T_{r}\right)$. Then $A: F \rightarrow F$ is a continuous and bounded operator. Now we prove that for any $H \subset F$, there exist a positive integer $n_{0}$ and $0<\gamma<1$ such that

$$
\alpha_{C}\left(A^{n_{0}}(H)\right) \leq \gamma \alpha_{C}(H)
$$

where $A^{n}(H)=A\left(\bar{c} o\left(A^{n-1}(H)\right)\right), n=2,3, \ldots$ By the definition of the operator $A$ and the assumption that $f$ is uniformly continuous, $A(H)$ are bounded and equicontinuous on $J$. Hence from Lemma 2.4, $A^{n}(H)$ $(n=1,2, \ldots)$ are bounded and equicontinuous. Therefore, for any positive integer $n$, we get from Lemma 2.1

$$
\alpha_{C}\left(A^{n}(H)\right)=\sup _{t \in J} \alpha\left(\left(A^{n}(H)\right)(t)\right) .
$$

It follows from (3.2), (3.4) and Lemma 2.2

$$
\begin{aligned}
\alpha((A H)(t)) & \leq \alpha\left(\int_{0}^{a} W(t, s) f(s, H(s), T H(s),(S H)(s)) d s\right) \\
& \leq \int_{0}^{a} W(t, s)\left[L_{1}(s)+s k_{0} L_{2}(s)+a h_{0} L_{3}(s)\right] d s \alpha(H) \\
& \leq L \sigma(t) \alpha(H),
\end{aligned}
$$

where $L=\int_{0}^{a} \omega(s)\left[L_{1}(s)+s k_{0} L_{2}(s)+a h_{0} L_{3}(s)\right] d s$. Set

$$
\alpha\left(\left(A^{k} H\right)(t)\right) \leq L \rho^{k-1} \sigma(t) \alpha(H),
$$

we have

$$
\alpha\left(\left(A^{k+1} H\right)(t)\right) \leq \alpha\left(\int_{0}^{a} W(t, s) f\left(s, A^{k} H(s), T A^{k} H(s),\left(S A^{k} H(s)\right) d s\right)\right.
$$




$$
\begin{aligned}
& \leq \int_{0}^{a} W(t, s)\left[L_{1}(s)+s k_{0} L_{2}(s)+a h_{0} L_{3}(s)\right] \alpha\left(A^{k} H(s)\right) d s \\
& \leq L \rho^{k} \sigma(t) \alpha(H) .
\end{aligned}
$$

By the mathematical reduction, we obtain

$$
\alpha_{C}\left(\left(A^{n}(H)\right)\right) \leq L \rho^{n-1} \max _{t \in J} \sigma(t) \alpha_{C}(H), \quad n=1,2,3, \ldots
$$

Thus, there exist a positive integer $n_{0}$ and $0<\gamma<1$ such that

$$
\alpha_{C}\left(\left(A^{n_{0}}(H)\right)\right) \leq \gamma \alpha_{C}(H),
$$

which imply that the operator $A$ has at least one fixed point $u \in H \subset F$ $\subset C(J, E)$ from the Lemma 2.3. So the integral equation (1.1) has at least one solution $u \in C(J, E)$.

(H4). For any $r>0, f$ is bounded and uniformly continuous on $J \times B_{r} \times B_{r} \times B_{r}$, and there exist non-negative Lebesgue integrable functions $L_{i}(i=1,2,3)$ such that for any $x_{i}, y_{i} \in E(i=1,2,3)$ and $t \in J$,

$$
\left\|f\left(t, y_{1}, y_{2}, y_{3}\right)-f\left(t, x_{1}, x_{2}, x_{3}\right)\right\| \leq \sum_{i=1}^{3} L_{i}(t)\left\|y_{i}-x_{i}\right\| .
$$

Theorem 3.2. Let $E$ be a real Banach space, the assumptions (H3), (H4) be satisfied. Then integral equation (1.1) has an unique solution $u \in C(J, E)$. Moreover, for any $z_{0} \in C(J, E)$, the iterative sequence defined by

$$
z_{n}(t)=\psi(t)+\int_{0}^{a} W(t, s) f\left(s, z_{n-1}(s),\left(T z_{n-1}\right)(s),\left(S z_{n-1}\right)(s)\right) d s, \quad n=1,2,3, \cdots,
$$

converges to $u^{*}(t)$ uniformly as norm $\|\cdot\|_{C}$ on $J$ and the error estimate is 


$$
\left\|u^{*}-z_{n}\right\| \leq L \max _{t \in J} \sigma(t) \cdot \frac{\rho^{n-1}}{1-\rho}\left\|A z_{0}-z_{0}\right\|
$$

where the constants $L, \rho$ and the function $\sigma$ are defined in Theorem 3.1.

Proof. From (3.3), for any $x, y \in C(J, E)$, by the similar rational procedure of (3.9), we have

$$
\left\|\left(A^{n} x\right)(t)-\left(A^{n} y\right)(t)\right\| \leq L \rho^{n-1} \sigma(t)\|x-y\|, \quad t \in J, \quad n=1,2,3, \ldots,
$$

and then

$$
\left\|\left(A^{n} x\right)-\left(A^{n} y\right)\right\| \leq L \rho^{n-1} \max _{t \in J} \sigma(t)\|x-y\|
$$

For any $z_{0} \in C(J, E)$, let $x=A z_{0}, y=z_{0}$. And from (3.12), we have for any positive integer $p>0$

$$
\begin{aligned}
\left\|z_{n+p}-z_{n}\right\| & \leq\left\|z_{n+p}-z_{n+p-1}\right\|+\left\|z_{n+p-1}-z_{n+p-2}\right\|+\cdots+\left\|z_{n+1}-z_{n}\right\| \\
& \leq L \max _{t \in J} \sigma(t)\left(\rho^{n+p-2}+\rho^{n+p-3}+\cdots+\rho^{n-1}\right)\left\|A z_{0}-z_{0}\right\| \\
& \leq \frac{\rho^{n-1}}{1-\rho} L \max _{t \in J} \sigma(t)\left\|A z_{0}-z_{0}\right\| .
\end{aligned}
$$

Thus from (3.3), $z_{n}$ is a Cauchy sequence in $C(J, E)$. Let $u^{*}=\lim _{n \rightarrow \infty} z_{n}$, from (3.4), we know $u^{*}$ is a solution of (1.1). Moreover, for (3.13), let $p \rightarrow \infty$, we have the error estimate formula (3.11).

On the other hand, if $u^{* *}$ is also the solution of (1.1), i.e., $u^{* *}=A u^{* *}$, then from (3.12), we have, as $n \rightarrow \infty$,

$$
\left\|u^{* *}-z_{n}\right\|=\left\|A^{n} u^{* *}-A^{n} z_{0}\right\| \leq L \max _{t \in J} \sigma(t) \rho^{n-1}\left\|u^{* *}-z_{0}\right\| \rightarrow 0 .
$$

This imply $u^{* *}=u^{*}$ and the theorem is proved. 


\section{Application in Three-Point Boundary Value Problems}

The $\mathrm{BVP}(1.2)$ is equivalent to the nonlinear integro-differential equation

$$
u(t)=\psi(t)+\int_{0}^{1} G(t, s) f(s, u(s), T u(s),(S u)(s)) d s
$$

where $\psi(t)=0$,

$$
G(t, s)=\frac{1}{\tau} \begin{cases}s[1-b \mu-t(1-b)], & 0 \leq s \leq t \leq \mu<1 \text { or } 0 \leq s \leq \mu \leq t \leq 1, \\ t[1-b \mu-s(1-b)], & 0 \leq t \leq s \leq \mu<1, \\ s(1-b \mu)-t(s-b \mu), & 0<\mu \leq s \leq t \leq 1, \\ t(1-s), & 0<\mu \leq t \leq s \leq 1 \text { or } 0 \leq t \leq \mu \leq s \leq 1,\end{cases}
$$

and $\tau=1-b \mu$. Let

$$
w(s)=\frac{1}{\tau} \begin{cases}1-b \mu-s(1-b), & 0 \leq s \leq \mu<1, \\ 1-s, & 0<\mu \leq s \leq 1,\end{cases}
$$

easily we can see

$$
|G(t, s)| \leq w(s) t, \quad t, s \in[0,1]
$$

Thus, we get following conclusions from Theorem 3.1.

Theorem 4.1. If the assumptions $(\mathrm{H} 1)$, (H2) be satisfied and the inequality

$$
\rho=\int_{0}^{1} s w(s)\left[L_{1}(s)+s k_{0} L_{2}(s)+h_{0} L_{3}(s)\right] d s<1
$$

hold, then BVP(1.2) has at least one solution.

Theorem 4.2. If the assumption (H4) be satisfied and the inequality (4.1) hold, then $B V P(1.2)$ has an unique solution $u^{*}(t)$. Moreover, for any $z_{0} \in C(J, E)$, the iterative sequence defined by

$$
z_{n}(t)=\psi(t)+\int_{0}^{a} G(t, s) f\left(s, z_{n-1}(s),\left(T z_{n-1}\right)(s),\left(S z_{n-1}\right)(s)\right) d s, \quad n=1,2,3, \ldots
$$


converges to $u^{*}(t)$ uniformly as norm $\|\cdot\|_{C}$ on $J$ and the error estimate is

$$
\left\|u^{*}-z_{n}\right\| \leq L \cdot \frac{\rho^{n-1}}{1-\rho}\left\|A z_{0}-z_{0}\right\|
$$

where the constants $L=\max _{t \in J} \int_{0}^{1} w(s)\left[L_{1}(s)+s k_{0} L_{2}(s)+h_{0} L_{3}(s)\right] d s$ and $\rho$ is defined by the formula (4.1).

Proof. Since it is similar to the proof of Theorem 3.2, we omit the proof.

Especially, as the functions $L_{i}$ in (H1) fade into constants $l_{i}(i=1,2,3)$. Directly calculating the integral in the formula (4.1), we obtain that the constants $L$ and $\rho$ in the Theorems 4.1 and 4.2 can be replaced by the following formulas:

$$
\rho=\int_{0}^{1} s w(s)\left[l_{1}+s k_{0} l_{2}+h_{0} l_{3}\right] d s=\frac{2\left(l_{1}+h_{0} l_{3}\right)\left(1-b \mu^{3}\right)+k_{0} l_{2}\left(1-b \mu^{4}\right)}{12(1-b \mu)},
$$

and

$$
L=\int_{0}^{1} w(s)\left[l_{1}+s k_{0} l_{2}+h_{0} l_{3}\right] d s=\frac{3\left(l_{1}+h_{0} l_{3}\right)\left(1-b \mu^{2}\right)+k_{0} l_{2}\left(1-b \mu^{3}\right)}{6(1-b \mu)},
$$

which can be conveniently used in our Theorems 4.1 and 4.2 .

\section{References}

[1] Mircea Dan Rus, A note on the existence of positive solutions of Fredholm integral equations, Fixed Point Theory 5 (2004), 369-377.

[2] Ravi P. Agarwal, Said R. Grace and Donal O’Regan, Existence of positive solutions to semipositone Fredholm integral equations, Funkcialaj Ekvacioj 45 (2002), 223-235.

[3] F. Calio, A. I. Garralda-Guillem, E. Marchetti and M. R. Galan, Numerical approaches for systems of Volterra-Fredholm integral equations, Applied Mathematics and Computation 225 (2013), 811-821. 
[4] G. Q. Long and Gnaneshwar Nelakantib, Iteration methods for Fredholm integral equations of the second kind, Comput. Math. Appl. 53 (2007), 886-894.

[5] H. Y. Li and X. L. Wang, The existence of multiple solutions for a class of nonlinear Fredholm type integral equations, Chinese J. Eng. Math. 22 (2005), 909-913 (in Chinese).

[6] A. X. Qian and Z. Q. Zhao, Coupled quasi-solutions and solutions of nonlinear impulsive Fredholm integral equations in Banach spaces, J. Sys. Sci. \& Math. Scis. 24 (2004), 488-495 (in Chinese).

[7] H. B. Chen and P. L. Li, Three-point boundary value problems for second-order ordinary differential equations in Banach spaces, Comput. Math. Appl. 56 (2008), 1852-1860.

[8] Z. G. Wang, L. S. Liu and Y. H. Wu, The unique solution of boundary value problems for nonlinear second-order integral-differential equations of mixed type in Banach spaces, Comput. Math. Appl. 54 (2007), 1293-1301.

[9] L. S. Liu, L. L. Liu and Y. H. Wu, Positive solutions of two-point boundary value problems for systems of nonlinear second-order singular and impulsive differential equations, Nonlinear Anal. 69 (2008) 3774-3789.

[10] M. Q. Feng and H. H. Pang, A class of three-point boundary-value problems for second-order impulsive integro-differential equations in Banach spaces, Nonlinear Anal. 70 (2009), 64-82.

[11] K. Deiming, Nonlinear Functional Analysis, Springer-Verlag, Berlin, 1985.

[12] V. Lakshmikantham and S. Leela, Nonlinear Differential Equations in Abstract Spaces, Pergamon Press, New York, 1981.

[13] Dajun Guo, V. Lakshmikantham and Xinzhi Liu, Nonlinear Integral Equations in Abstract Spaces, Kluwer Academic Publisher, Dordrecht, 1996.

[14] L. S. Liu, F. Guo, C. X. Wu and Y. H. Wu, Existence theorems of global solutions for nonlinear Volterra type integral equations in Banach spaces, J. Math. Anal. Appl. 309 (2005), 638-649.

[15] L. S. Liu, Iterative method for solutions and coupled quasi-solutions of nonlinear Fredholm integral equations in ordered Banach spaces, Indian J. Pure Appl. Math. 27 (1996), 959-972. 